\title{
Strongly Enhanced Orbital Moments and Anisotropies of Adatoms on the $\operatorname{Ag}(001)$ Surface
}

\author{
B. Nonas, I. Cabria, R. Zeller, and P. H. Dederichs \\ Institut für Festkörperforschung, Forschungszentrum Jülich, D-52425 Jülich, Germany \\ T. Huhne and H. Ebert \\ Institut für Physikalische Chemie, Universität München, Butenandtstrasse 5-13, D-81377 München, Germany
}

(Received 31 August 2000)

\begin{abstract}
We present $a b$ initio calculations for orbital moments and anisotropy energies of $3 d$ and $5 d$ adatoms on the $\mathrm{Ag}(001)$ surface, based on density functional theory, including Brooks' orbital polarization (OP) term, and applying a fully relativistic Korringa-Kohn-Rostoker-Green's function method. In general, we find unusually large orbital moments and anisotropy energies, e.g., in the $3 d$ series, $2.57 \mu_{B}$ and $+74 \mathrm{meV}$ for Co, and, in the $5 d$ series, $1.78 \mu_{B}$ and $+42 \mathrm{meV}$ for Os. These magnetic properties are determined mainly by the OP and even exist without spin-orbit coupling.
\end{abstract}

PACS numbers: 75.70.Cn, 71.70.Ej, 75.30.Gw

The interest in surface magnetism is primarily caused by the enhancement of the spin moments at surfaces being driven by the reduced coordination. A typical example for this effect is iron, for which the bulk moment $\left(2.15 \mu_{B}\right)$ is enhanced at the (001) surface to $2.9 \mu_{B}$ [1]. Similar enhancements are also found in ultrathin magnetic films [2] and in particular in $3 d$ monolayers on noble metal surfaces [3] where the $3 d$ moments approach the atomic values given by Hund's first rule. Also some $4 d$ monolayers seem to be magnetic. For example, for $\mathrm{Rh}$ and $\mathrm{Ru}$ on a $\mathrm{Ag}$ or $\mathrm{Au}$ surface, moments of about 0.62 and $0.29 \mu_{B}$, respectively, have been calculated [4]. Even larger moments have been obtained in ab initio calculations for single transition metal adatoms on the surfaces of $\mathrm{Cu}, \mathrm{Ag}, \mathrm{Au}$ [5], or $\mathrm{Pd}$ and $\mathrm{Pt}$ [6]. Here the $4 d$ and $5 d$ atoms, being nonmagnetic as impurities in the bulk, show as adatoms very large local moments comparable with the free atom values. Sizable moments also survive when these atoms are incorporated into the first layer.

In contrast to the spin magnetism, the orbital magnetism in solids has its origin in the spin-orbit interaction and is closely connected with the magnetocrystalline anisotropy, with magneto-optical effects and magnetic $\mathrm{x}$-ray dichroism. In this paper we address the enhancement of orbital moments at surfaces. It is well known that the orbital moments are "quenched" in the bulk, i.e., strongly suppressed by the crystal field splitting, the strong hybridization with the neighboring atoms, respectively. Thus, calculations yield very small orbital moments: $0.049 \mu_{B}, 0.075 \mu_{B}$, and $0.042 \mu_{B}$ for bcc Fe, hep Co, and fcc Ni $\left[0.082 \mu_{B}, 0.123 \mu_{B}\right.$, and $0.058 \mu_{B}$ if Brooks' orbital polarization (OP) is included] [7]. At surfaces $a b$ initio calculations show that also the orbital moments are enhanced, to e.g., a value of $0.090 \mu_{B}$ at the hcp $\operatorname{Co}(0001)$ surface $\left(0.158 \mu_{B}\right.$ if Brooks' OP is included) $[7,8]$. Even larger orbital moments are obtained for the $3 d$ monolayers, e.g., $0.121 \mu_{B}$ for a Co monolayer on $\mathrm{Cu}(100)\left(0.261 \mu_{B}\right.$ including OP) [7,9]. Thus, at surfaces the quenching of the orbital moments is less pronounced due to the reduced hybridization. However, it is important to realize that these enhanced orbital moments are still an order of magnitude smaller than the corresponding free atom values, as given by Hund's second rule. Thus, the orbital moments are to a large extent also quenched at the surface, as it seems to be the general rule in metallic environments.

However, this rule can have exceptions. Riegel and co-workers [10] have already shown that Fe impurities, being injected into alkali metals, show hyperfine properties which indicate very large orbital moments, probably the full atomic values. The low and more or less constant electron density of the alkali hosts is responsible for this behavior. Here we will predict by density functional calculations that single $3 d$ and $5 d$ transition metal impurities on the $\operatorname{Ag}(001)$ surface can have very large orbital moments and extremely large magnetic anisotropy energies.

Our investigations have been carried out within the framework of the relativistic version of spin-densityfunctional theory. The corresponding Dirac-Kohn-Sham equation for the four-component single-particle Green's function $G(E)$ is solved within the local density approximation for spin-polarized spherical potentials using the KKR-Green's function formalism. Similar to the bulk, the vacuum region is described by spherical potentials at "empty" fcc lattice sites, and the adatom is placed at a regular fcc site in the first vacuum layer, i.e., the hollow site. Lattice relaxations are neglected.

The Green's function of the system is constructed from the regular and irregular solutions of the Dirac-Kohn-Sham equation as described in Ref. [8]. The corresponding scattering path operator $\tau_{\Lambda \Lambda^{\prime}}^{n n^{\prime}}$ is obtained via an algebraic Dyson equation

$$
\boldsymbol{\tau}_{\Lambda \Lambda^{\prime}}^{n n^{\prime}}=\stackrel{\circ}{\boldsymbol{\tau}}_{\Lambda \Lambda^{\prime}}^{n n^{\prime}}+\sum_{n^{\prime \prime} \Lambda^{\prime \prime} \Lambda^{\prime \prime \prime}} \stackrel{\circ}{\boldsymbol{\tau}}_{\Lambda \Lambda^{\prime \prime}}^{n n^{\prime \prime}}\left(\boldsymbol{t}^{-1}-\stackrel{\circ}{\boldsymbol{t}}-1\right)_{\Lambda^{\prime \prime} \Lambda^{\prime \prime \prime}}^{n^{\prime \prime}} \stackrel{\circ}{\boldsymbol{\tau}}_{\Lambda^{\prime \prime \prime} \Lambda^{\prime \prime}}^{n^{\prime \prime} n^{\prime}}
$$


which relates $\tau$ to the scattering path operator of a reference system $\%$ via the difference in the respective single site scattering matrices $t$. By assuming a finite extension of the perturbation, Eq. (1) turns into a matrix equation which may be solved by inversion.

The orbital moments calculated in the relativistic LDA formalism are usually about $30 \%-50 \%$ smaller than the corresponding experimental values due to the neglect of correlation effects. Brooks has suggested a way to improve this shortcoming by an additional heuristic term in the Hamiltonian [11,12], which we include in our calculations for the $d$ valence electrons in the form suggested by Ebert [13]:

$$
H_{s}^{\mathrm{OP}}=-B_{s}^{\mathrm{OP}}(r)\left\langle\hat{l}_{z}^{s}\right\rangle \hat{l}_{z} \delta_{s, s^{\prime}} \delta_{l, 2},
$$

where $B_{s}$ denotes the corresponding Racah parameter and $s= \pm 1 / 2$ is the quantum number corresponding to $s_{z}$. This operator acts only on the states with $l=2$ and the same quantum number $s$.

One central result of the present paper is shown in Fig. 1. The local spin and orbital moments for the $3 d$ and $5 d$ atoms as adsorbate atoms on the $\operatorname{Ag}(001)$ surface have been calculated self-consistently in the spin-polarized relativistic Dirac scheme, both by including (SPR-OP) or excluding (SPR) the OP term. For comparison, the spin moments have also been calculated in the scalar relativistic approximation (SRA). First, we notice that the $3 d$ spin moments are very large and agree very well for all three schemes. In fact, in the SPR-OP treatment the spin moments are, in general, slightly smaller than for the SRA. However, for the $3 d$ atoms the differences are smaller than $10^{-2} \mu_{B}$, except for $\mathrm{Ni}$, and cannot be seen in the figure. The spin moments of the $5 d$ adatoms are considerably smaller than the $3 d$ ones due to the larger extent of the $5 d$ wave functions and the resulting stronger hybridization with the Ag substrate electrons. Nevertheless, these moments show the same parabolic behavior with the valence of the adatoms. Here we see that in the fully relativistic treatment the $5 d$ spin moments are slightly reduced. For instance, for Os the SRA moment of $2.42 \mu_{B}$ is reduced to $2.06 \mu_{B}$ in the SPR scheme, while the SPR-OP scheme reduces this further to $2.04 \mu_{B}$. Most significant is the re- duction for Ir, for which the moment vanishes in a relativistic treatment. Qualitatively, the reduction of the spin moments can be understood from the broadening of the local density of states (LDOS) due to the spin-orbit splitting and the splitting induced by the OP. Because of the larger spin-orbit coupling (SOC) parameter these effects are larger for the $5 d$ atoms.

In addition to the spin moments, Fig. 1 also shows the orbital moments of the adatoms, calculated selfconsistently in the fully relativistic (SPR) scheme (dotted line) and in the SPR-OP scheme with Brooks' OP term (full line). For the $3 d$ and $5 d$ atoms we observe a change of the orbital moment from negative values in the first half of the series to positive values in the second half, with reduced values at the beginning and at the end of the series. The enhancement of the orbital moments by the OP varies between $30 \%$ to $40 \%$, except for $\mathrm{Fe}$, Co, and $\mathrm{Ni}$ adatoms, where this amounts to a factor bigger than 3 . Most important is the observation that, with the exception of $\mathrm{Fe}$ and $\mathrm{Co}$ adatoms, the $5 d$ orbital moments are considerably larger than the $3 d$ ones. This is a consequence of the much larger SOC parameter of the $5 d$ transition metal atoms, which strongly influences the orbital moments (see below). In the $3 d$ series, the by far largest orbital moments are obtained for $\mathrm{Fe}$ and $\mathrm{Co}$ adatoms, with values of $2.20 \mu_{B}$ and $2.57 \mu_{B}$ in the SPR-OP scheme and $0.55 \mu_{B}$ and $0.76 \mu_{B}$ in the relativistic scheme without OP. To our knowledge, these are the largest orbital moments which have been obtained in ab initio calculations for transition metals, their metallic alloys and surfaces. This is a direct consequence of the exposed position on top of the surface. Except for $\mathrm{Fe}, \mathrm{Co}$, and $\mathrm{Ni}$, these values are, however, still considerably smaller than the atomic orbital moments.

In order to clarify the roles of SOC and OP, it is instructive to analyze the local density of states (LDOS) of the adatoms. For the two elements with the largest orbital moments, the $3 d$ adatom Co and the $5 d$ adatom Os, the spin-resolved LDOS are shown in Fig. 2, where the full line (SPR-OP) gives the relativistic results including the OP term and the dashed line (SPR) the ones without OP. In the SRA one obtains two relatively sharp virtual bound states being separated by the exchange splitting IM, where
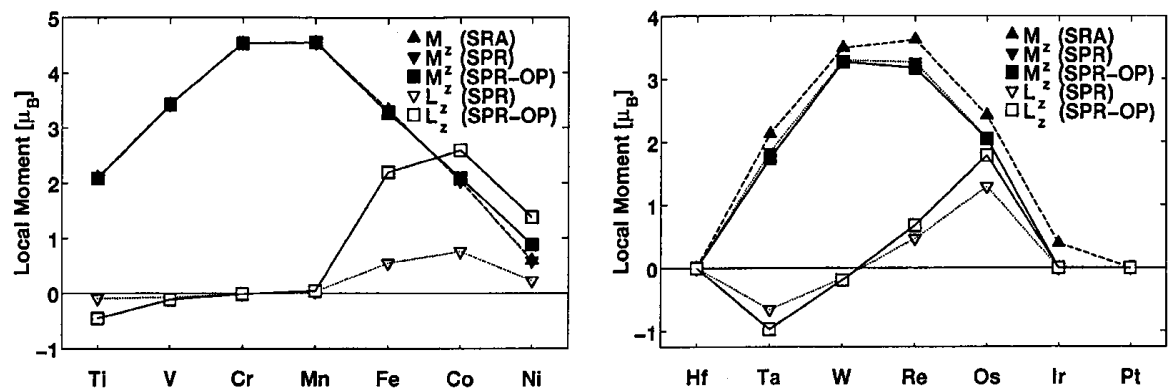

FIG. 1. Calculated spin and orbital moments of the $3 d$ adatoms (left) and $5 d$ adatoms (right) on the Ag(001) surface. The upper two curves show the spin moments in the SRA (dashed lines, $\boldsymbol{\Delta}$ ) and the SPR-OP scheme (full lines, $\mathbf{\square}$ ). The two lower curves show the orbital moments in the SPR-OP (full lines, $\square$ ) and the SPR scheme (dotted lines, $\nabla$ ). 

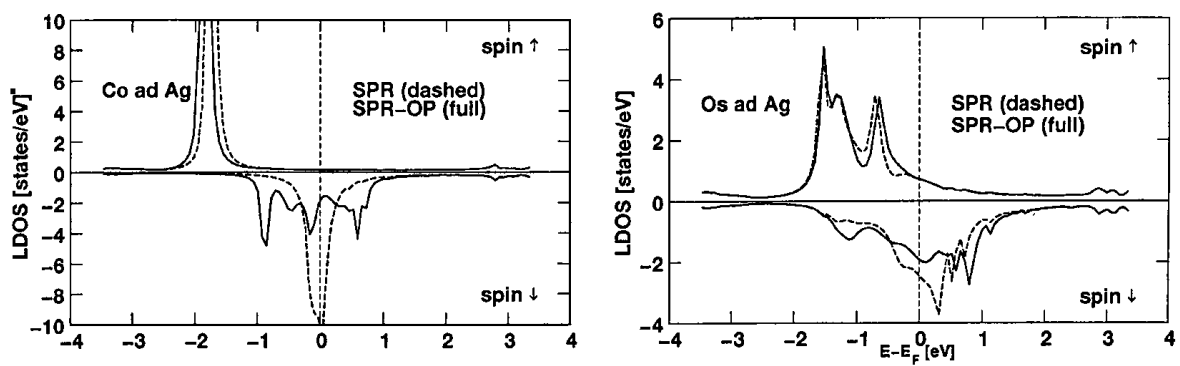

FIG. 2. Local density of states of the adatoms Co and Os on the $\operatorname{Ag}(001)$ surface, obtained in SPR and SPR-OP calculations.

I is the exchange integral and $\mathrm{M}$ the local moment. These peaks are split up by both the SOC and the OP. Since the SOC is much smaller than the exchange splitting IM, the spin-orbit operator $\vec{l} \cdot \vec{s}$ can be replaced by $l_{z} s_{z}$, so that spin-flip processes can be neglected and the spin is still a good quantum number. Thus, the SOC splits up the virtual bound state for each spin direction into 5 subpeaks with magnetic quantum numbers $m=-2,-1,0,+1,+2$, being separated by $\frac{\xi}{2}$, where $\xi$ is the SOC parameter, which is about $0.06 \mathrm{eV}$ for $\mathrm{Co}$ and $0.5 \mathrm{eV}$ for Os. Thus, as expected, the spin-orbit splitting is small for Co and larger for Os. The above discussion assumes that the crystal field splitting is small and can be neglected, which is reasonably well satisfied in both cases. The OP term leads to an additional $m$ splitting, with spin dependent peak splittings given by $B_{s}^{\mathrm{OP}}\left\langle\hat{l}_{z}^{s}\right\rangle$. Since the majority band is filled and has no orbital moment $\left(\left\langle\hat{l}_{z}^{\dagger}\right\rangle=0\right)$, only the minority bands are affected by these splittings $\left(B_{\downarrow}^{\mathrm{OP}}=0.14\right.$ and $0.10 \mathrm{eV}$ for Co and Os, respectively). Thus, we see that for Co the OP splitting is much larger than the SOC splitting.

The large OP splittings of the LDOS as well as the large OP enhancements of the orbital moments of $\mathrm{Fe}, \mathrm{Co}$, and Ni (Fig. 1) suggest that basically the OP term determines the orbital moments and the SOC is of secondary importance. Of course, this is the expected behavior in the atomic limit as described by Hund's second rule. For this reason, we have manipulated the strength of the SOC in the Dirac-Kohn-Sham equation as described in Ref. [14]. Even for vanishing SOC, i.e., in the SRA limit with the OP term included, we obtain essentially the same results as with SOC and OP included. The LDOS is practically identical to the solid SPR-OP lines in Fig. 2 and the orbital moments are only slightly reduced, e.g., for Co from 2.57 in SPR-OP to $2.40 \mu_{B}$ in SRA-OP, for Os from $1.78 \mu_{B}$ to $1.75 \mu_{B}$. Thus, we obtain a "spontaneous" orbital moment which does not need the induction by the SOC. This is fully in line with the argument in Ref. [15]. Because of the high LDOS at $E_{F}$ in the minority band, the Stoner-like criterium for the occurrence of a spontaneous orbital moment is satisfied in the SRA treatment, i.e., $\sum_{m=-l}^{l} m^{2} n_{2, m}^{\downarrow}\left(E_{F}\right) B_{\downarrow}^{\mathrm{OP}}>1$, where $m$ and $n_{2, m}^{\downarrow}\left(E_{F}\right)$ are the magnetic quantum number and the $m$ resolved spin down SRA-LDOS for $l=2$ without OP. This is clearly a consequence of the pronounced peak of the minority vir- tual bound state at $E_{F}$, which is typical for the considered impurities $\mathrm{Fe}, \mathrm{Co}$, and Os. This situation usually does not occur in metallic systems, since, due to band formation, the LDOS at $E_{F}$ is much lower. Then, the SOC induces the orbital moment, in much the same way as a magnetic field induces a spin moment in a nonmagnetic system, and the OP term enhances it [15], similar to the Stoner factor enhancing the spin moment.

Unfortunately the OP effect in solids is not well understood at present [16]. In particular, the accuracy of Brooks approximation is to some extent uncertain, especially when applied to nearly localized systems as the magnetic adatoms in this paper. Therefore, improved calculations based, e.g., on the LDA+U method [16], the LDA-SIC approach [17], or improved current density functionals [18] would be most helpful. However, we believe that the qualitative results obtained in this paper, in particular the strong enhancement of orbital moments and anisotropy energies (see below) as well as the strong importance of the OP, are generally valid.

The magnetic anisotropy energy (MAE) as the energy difference between the easy and hard axis orientation of the magnetic moment is particularly large for surfaces and layered systems since, as a consequence of the symmetry reduction, it scales quadratically with the SOC parameter $\xi$. Bruno [19] has pointed out a direct relation of the MAE to the anisotropy of the orbital moment, and a more rigorous formula has been recently derived by van der Laan [20]. For these reasons one might expect that the large orbital moments of the magnetic adatoms are also connected with large MAEs. The calculated results for the MAEs of the adatoms are shown in Fig. 3, both for the $3 d$ adatoms (left) and the $5 d$ ones (right). Positive values mean an orientation perpendicular to the surface, negative ones an in-plane orientation. First, we notice that the calculated MAEs are indeed very large. In particular, the huge values for the $5 d$ impurities $\mathrm{Re}$ and $\mathrm{Os},-38$ and $+42 \mathrm{meV}$, respectively, are presumably the largest values ever obtained for transition metal systems. The even larger values for $\mathrm{Co}$ and $\mathrm{Ni}$, i.e., +74 and $+53 \mathrm{meV}$, are practically only determined by the OP. Second, we notice that the anisotropy energy varies oscillatory with the valence of the impurities and that, in particular, at the end of the series the perpendicular orientation is preferred. The 

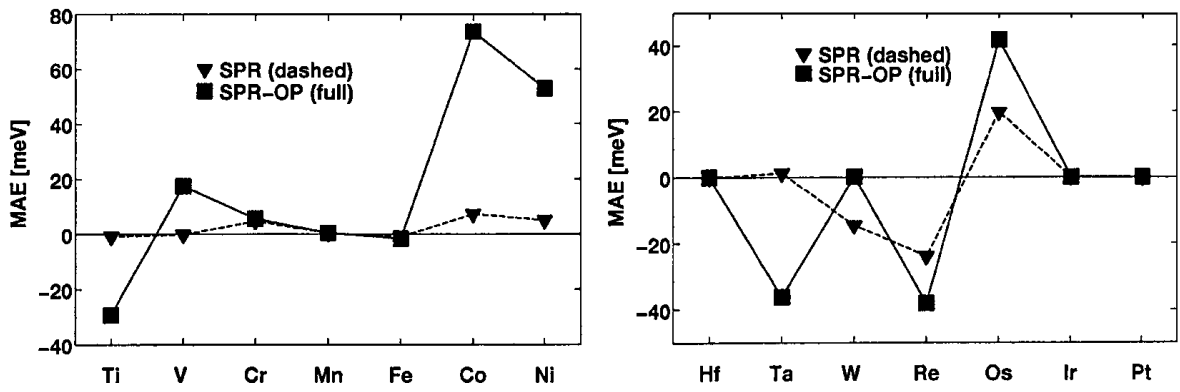

FIG. 3. Magnetic anisotropy energy for $3 d$ and $5 d$ adatoms on the $\operatorname{Ag}(001)$ surface, obtained in SPR and SPR-OP calculations.

oscillatory dependence has also been found in several calculations for monolayers [21], and can basically be traced back to a general theorem by Heine et al. [22]. While we cannot give more details of the calculations, we notice that the MAE of the $5 d$ metals cannot be calculated by a perturbative treatment like, e.g., the force theorem since the two configurations involved, magnetic moments perpendicular and parallel to the surface, are considerably different.

In summarizing, we have performed fully relativistic calculations based on the local density approximation with orbital polarization for $3 d$ and $5 d$ magnetic adatoms on the $\operatorname{Ag}(001)$ surface. The calculations predict unusually large orbital moments for the adatoms, with the largest values for Co, $2.57 \mu_{B}$, in the $3 d$ series, and for Os, $1.78 \mu_{B}$, in the $5 d$ series. This behavior is a consequence of the reduced coordination and the weak hybridization of the adatoms with the Ag substrate. The size of the moments is in the largest part determined by the OP.

Thus, these adatoms might be considered as providing a link between free atoms, which have fully developed orbital moments as given by Hund's rules, and atoms in the bulk or at clean surfaces, where only small orbital moments occur, being induced by spin-orbit coupling. The large orbital moments of the adatoms are connected with equally large magnetic anisotropy energies, representing the largest values known to us for transition metal systems. We hope that our results will encourage experimental efforts to search for these properties, e.g., by magnetic $\mathrm{x}$-ray dichroism. Also the large moments and anisotropies should encourage theoretical studies of small magnetic nanostructures with reduced coordinations.

We thank Stefan Blügel for helpful discussions concerning the magnetic anisotropy energy. This work was partially funded by the Schwerpunktprogramm "Relativistische Effekte" of the Deutsche Forschungsgemeinschaft and the TMR network "Interface Magnetism" of the European Union.

[1] S. Ohnishi, A. Freeman, and M. Weinert, Phys. Rev. B 28, 6741 (1983).

[2] S. Blügel, B. Drittler, R. Zeller, and P. Dederichs, J. Appl. Phys. A 49, 547 (1989).

[3] S. Blügel and P.H. Dederichs, Europhys. Lett. 9, 597 (1989).

[4] O. Eriksson, R. Albers, and A. Boring, Phys. Rev. Lett. 66, 1350 (1991).

[5] P. Lang et al., Solid State Commun. 92, 755 (1994).

[6] V. Stepanyuk et al., Phys. Rev. B 53, 2121 (1996).

[7] O. Hjortstam et al., Phys. Rev. B 53, 9204 (1996).

[8] T. Huhne et al., Phys. Rev. B 58, 10236 (1998).

[9] M. Tischer et al., Phys. Rev. Lett. 75, 1602 (1995).

[10] D. Riegel et al., Phys. Rev. Lett. 57, 388 (1986).

[11] M. Brooks, Physica (Amsterdam) 130B, 6 (1985).

[12] O. Eriksson, M. Brooks, and B. Johansson, Phys. Rev. B 41, 7311 (1990).

[13] H. Ebert and M. Battocletti, Solid State Commun. 98, 785 (1996).

[14] H. Ebert, H. Freyer, A. Vernes, and G.-Y. Guo, Phys. Rev. B 53, 7721 (1996).

[15] L. Nordström, Ph.D. thesis, Uppsala University, 1991.

[16] I. V. Solovyev, A. I. Liechtenstein, and K. Terakura, Phys. Rev. Lett. 80, 5758 (1998).

[17] S. V. Beiden, W. M. Temmerman, Z. Szotek, and G. A. Gehring, Phys. Rev. Lett. 79, 3970 (1997).

[18] H. Ebert, M. Battocletti, and E. K. U. Gross, Europhys. Lett. 40, 545 (1997).

[19] P. Bruno, Phys. Rev. B 39, 865 (1989).

[20] G. van der Laan, J. Phys. Condens. Matter 10, 3239 (1998).

[21] S. Pick and H. Dreyssé, Phys. Rev. B 46, 5802 (1992).

[22] V. Heine and J. H. Samson, J. Phys. F 13, 2155 (1983). 